\title{
Multisource Fusion/Classification Using ICM and DSmT with New Decision Rule
}

\author{
Azeddine Elhassouny ${ }^{1}$, Soufiane Idbraim ${ }^{1}$, Aissam Bekkarri ${ }^{1}$, \\ Driss Mammass ${ }^{1}$, and Danielle Ducrot ${ }^{2}$ \\ ${ }^{1}$ IRF-SIC Laboratory, Faculty of Science, Agadir, Morocoo \\ \{info_azeddine, soufianeidbraim, a_bekkari\}@yahoo.fr, \\ mammass@uiz.ac.ma \\ ${ }^{2}$ CESBIO Laboratory Toulouse, France
}

\begin{abstract}
In this paper we introduce a new procedure for classification and change detection by the integration in a fusion process using hybrid DSmT model, both, the contextual information obtained from a supervised ICM classification with constraints and the temporal information with the use of two images taken at two different dates. Secondly, we have proposed a new decision rule based on the DSmP transformation, which is as an alternative and extension and overcoming the inherent limitations of the decision rules thus use the maximum of generalized belief functions.
\end{abstract}

The approach is evaluated on two LANDSAT ETM+ images, the results are promising.

Keywords: Detection of the changes, Image classification, Fusion, Hybrid DSmT model, Decision rule, DSmP, Satellite images, ICM.

\section{Introduction}

The management and the follow-up of the rural areas evolution are one of the major concerns for country planning. The satellite images offer a rapid and economic access to accurate homogeneous and updated information of studied territories. An example of application which results from this is related to the topic of the changes cartography, in this paper, we are interested to study the most subtle changes of the Argan land cover and other themes in the region of Agadir (Morocco) by contextual fusion /classification multidates based on hybrid DSmT model [1-3] and ICM with constraints $[4,5]$.

Our work environment, is the theory of Dezert-Smarandache [1-3] which is recent and very little implemented or used before the covered work of this paper, it was applied in multidate fusion for the short-term prediction of the winter land cover [6-9] and, recently, for the fusion and the multidate classification [10-12], although the theory of evidence, it is more exploited for fusion/classification [12-18] also, for classifier fusion [19-20].

Our methodology can be summarized as following, after preprocessing of the images, a supervised ICM classification with constraints [4, 5] is applied to the two 
images, in order to recover the probabilities matrices for an after using in a step of fusion/classification basing on the theory of plausible and paradoxical reasoning known as Dezert-Smarandache Theory (DSmT) which allows to better assign the suitable pixels to the appropriate classes and also to detecte the changes.

In this paper, in section 2, we describe the mathematical basis of the recent theory of plausible and paradoxical reasoning (DSmT), and give a description of our decision rule. In section 3, we provide the results of our experimentation where the algorithm was applied to a LANDSAT ETM+ image, the classification results are discussed in the same section followed by conclusions in section 4 .

\section{Dezert - Smarandache Theory (DSmT)}

\subsection{Principles of the DSmT}

The DSm theory was conceived jointly by Jean Dezert and Florentin Smarandache [1-3], it is a new way of representing and fusioning uncertain information. DSmT, considered as a generalization of the evidence theory of Demspter-Shafer [14], was developed to overcome the inherent limitations of DST (Dempster-Shafer Theory) [1-3]. The basic idea of DSmT rests on the definition of the hyper power set $\mathrm{D}^{\Theta}$ from elements of $\Theta$ with $\cup$ and $\cap$ operators. From which the mass functions, the combination rules and the generalized belief functions are built.

We define a map as follows:

$$
m_{s}(.): D^{\Theta} \rightarrow[0,1]
$$

Associated to a given body of evidence $s$ as

$$
m_{s}(\phi)=0
$$

and

$$
\sum_{X \in D^{\Theta}} m_{s}(X)=1
$$

with $m_{s}(X)$ is called the basic belief assignment/mass (bba) of $X$ made by the source $s$.

The DSmT contains two models : the free model and the hybrid model [1-3], the first presents limits concerning the size of the hyper power set $\mathrm{D}^{\Theta}$, whereas the second has the advantage of minimizing this size, for this reason, it will be used in the continuation of our study.

Within the framework of DSmT, there are several rules combination [1-3], we have applied and implemented the majority of these rules in order to choose those which allow us to have good performances such as the Proportional Conflict Redistribution (PCR5).

Mainly, the PCR5 rule is based on the principle of the (total or partial) conflicting masses redistribution [1-3] to the non-empty sets involved in the conflicts proportionally with respect to their masses assigned by the sources (it can be also generalized for $\mathrm{N}>2$ sources) [2,3]. 
The generalized belief functions used in this study namely the Credibility $(\mathrm{Cr})$, Plausibility (Pl) and DSmP Transformation [3] are defined for $\mathrm{D}^{\Theta}$ in [0,1] and are given with more detail in [1-3].

\subsection{Proposed Decision Rule}

The decisions after combinations could be taken from the basic belief assignment/mass (bba) or the generalized belief functions (Credibility (Cr), Plausibility (Pl), (DSmP) transformation $\cdots$ etc), thus, to decide the belonging of a pixel to a given class, two cases are distinguished:

- The pixel belonging to a simple class (used to improve a classification): in this case we use one of the following decision criteria: maximum of bba, maximum of the Credibility $(\mathrm{Cr})$ (with or without rejection), maximum of the Plausibility ( $\mathrm{Pl})$, Appriou criterium, DSmP criterium $\cdots$ etc) $[6,7,11,13,15]$.

- The pixel belonging to a composed class(e.g. in the case of change detection), in this case we cannot use the functions quoted previously because they are increasing functions and unsuited to the decision for the elements of union and of intersection.

In an original step, we have proposed a new decision rule based on DSmP transformation and confidence interval to take in account the composed classes. In this decision rule we exploited the confidence interval $[\operatorname{Bel}(\mathrm{X}), \mathrm{Pl}(\mathrm{X})]$ by the definition of a new measurement which we have named: Global uncertainty IncG which is the sum of the uncertainties (Inc) of the hyper power set elements:

$$
\forall X \in D^{\Theta} \operatorname{Inc}(X)=\operatorname{Pl}(X)-\operatorname{Bel}(X)
$$

This new decision rule described in Algorithm below is applied as follows:

For a given pixel x, we compare the Global uncertainty $\operatorname{Inc} G$ of this pixel with a threshold (chosen experimentally). If it is lower than this threshold, the pixel is affected to the simple class which maximizes the DSmP transformation of all the simple classes, if not; it is affected to the composed class which maximizes the mass (bba) of all the composed classes.

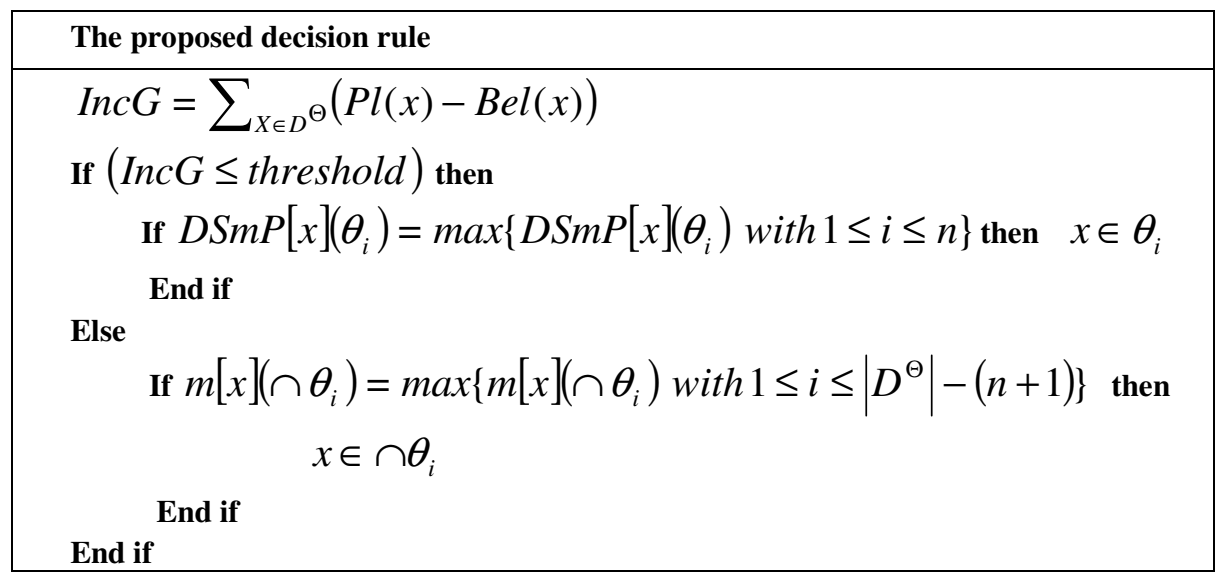




\section{Result and Discussion}

\subsection{Study Area and Used Data}

The study area is located in the region of Souss, it is in southern Morocco. Geologically, it is the alluvial basin of the Oued Souss, separated from the Sahara by the Anti-Atlas mountains. The natural vegetation in the Souss is savanna dominated by the Argan (Argania spinosa), a local endemic tree found nowhere else, part of the area is now a UNESCO Biosphere reserve to protect this unique habitat.

The satellite images used in this study were taken respectively, in 19 March 2002 and 12 April 2005 by the Landsat ETM+, and are defined by the coordinates (Path 203, Row 39).
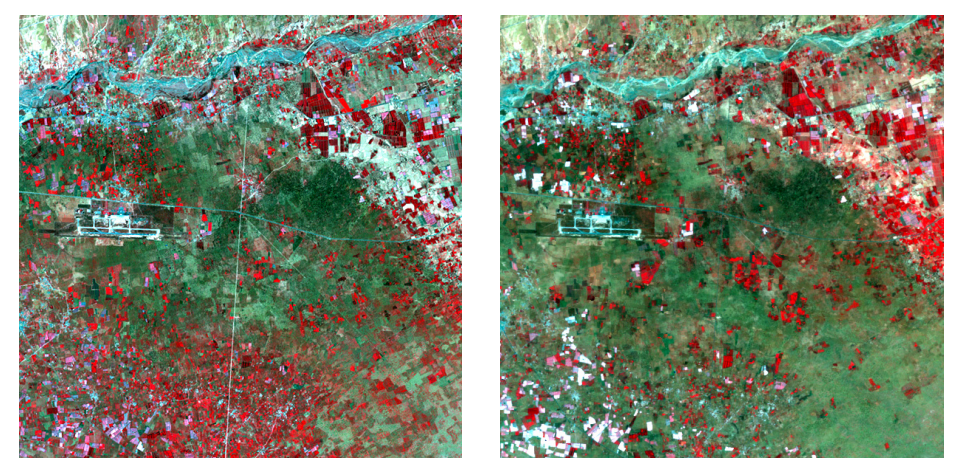

Fig. 1. RGB composite of the Landsat ETM+ images 2002 and 2005 (Agadir, Morocoo)

\subsection{ICM Classification with Constraints}

After preprocessing of the two images and establishment of the samples trainings (Argan (A), Built/Oued (BO), Vegetation (V), Greenhouses (Gh) and Bareground $(\mathrm{Bg})$ ), a supervised contextual ICM classification with constraints $[4,5]$ is applied to the two images to have the probabilities of pixels belonging to classes. The classified image of 2002 is presented in figure 2 (a).

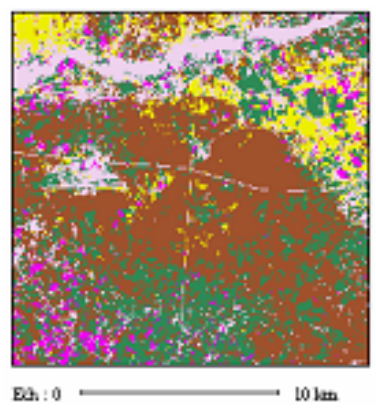

(a)
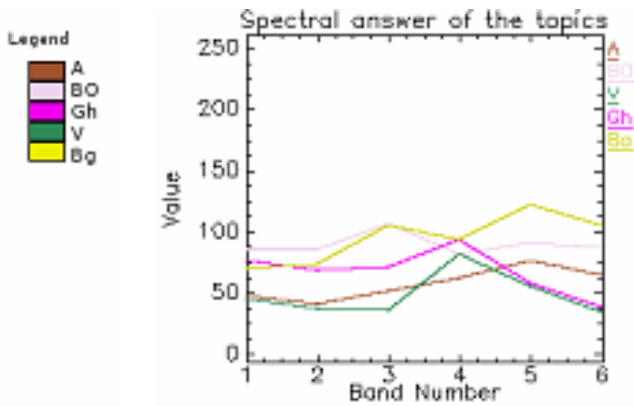

(b)

Fig. 2. ICM classification with constraints of the 2002 image (a) and Spectral response of the topics (b) 


\subsection{Multidates and Multi-source Fusion by the Hybrid DSmT Model}

Our fusion process is composed of the following steps, first the definition of the framework $\Theta$, then, the estimation of the mass functions of each focal element by the model of Appriou, finally, the application of the hybrid DSmT model.

\subsubsection{Hyper Power Set}

Taking in consideration the prior knowledge of the study area, we have identified 5 classes constituting the framework $\Theta$ section 3.2, $\Theta$ is defined as follows: $\Theta=\{\mathrm{A}, \mathrm{BO}$, $\mathrm{Gh}, \mathrm{V}, \mathrm{Bg}\}$, some elements of the hyper power set $\mathrm{D}^{\Theta}$ seem not being adjacents and exclusives. To realize a better adapted study to the real situations, some exclusivity

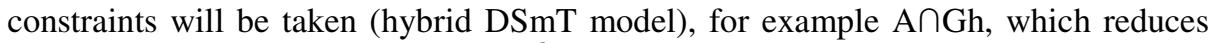
the number of focal elements of the $\mathrm{D}^{\Theta}$.

\subsubsection{Choice of Threshold}

The decision is made for the simple classes and the classes of intersection by using our rule decision defined previously (section2.2), the threshold should be determined in advance by experimentation and analysis of total uncertainty distribution after standardization. We have tested our decision rule with various values of threshold, the following table 1 presents the occupancy rates of the simple classes (stable zones) and composed classes (change zones) according to the threshold.

Table 1. Occupancy rates of the simple classes and the composed classes according to the value of the threshold

\begin{tabular}{lllllll}
\hline Threshold & $1.0 \mathrm{e}-26$ & $1.0 \mathrm{e}-019$ & $1.0 \mathrm{e}-016$ & $1.0 \mathrm{e}-014$ & $1.0 \mathrm{e}-012$ & $1.0 \mathrm{e}-08$ \\
\hline (\%) of stable zones & $0 \%$ & $9.64 \%$ & $32.12 \%$ & $54.408 \%$ & $76.86 \%$ & $99.99 \%$ \\
(\%)of change zones & $100 \%$ & $90.36 \%$ & $67.88 \%$ & $45.592 \%$ & $23.32 \%$ & $0.01 \%$ \\
\hline
\end{tabular}

The choice of threshold depends to the good results of detection with the use of the changed/ unchanged samples between the two dates (2002 and 2005), for this we have taken a suitable threshold that equal to 1.0e-014, which is coherent and appears to be close to reality basing on the test samples of the stable zones and the zones of changes between the two images.

\subsubsection{Validation of the Results}

3.3.3.1 Prevalidation of the Results. The fusion map obtained with an adequate threshold (1.0e-14) is presented in figure 3. 


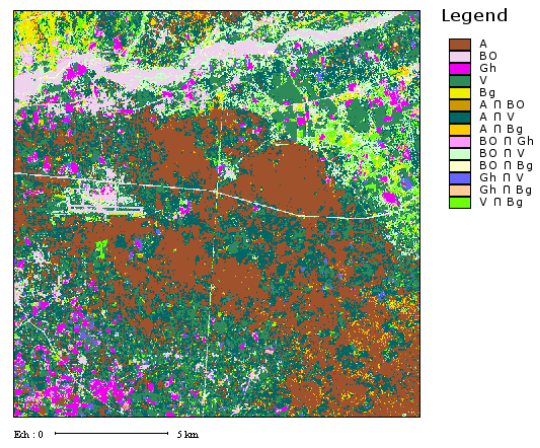

Fig. 3. Fusion map obtained with threshold of (1.0e-14)

From the fusion map, obtained with an adequate threshold we obtain the table 2 presenting the occupancy rate of the classes.

Table 2. Occupancy rate of the classes

\begin{tabular}{cccc}
\hline Class & $(\boldsymbol{\%})$ & Class & $(\boldsymbol{\%})$ \\
\hline $\mathrm{A}$ & $29.04 \%$ & $\mathrm{~A} \cap \mathrm{Bg}$ & $1.87 \%$ \\
$\mathrm{BO}$ & $4.869 \%$ & $\mathrm{BO} \cap \mathrm{Gh}$ & $1.549 \%$ \\
$\mathrm{Gh}$ & $3.18 \%$ & $\mathrm{BO} \cap \mathrm{V}$ & $7.25 \%$ \\
$\mathrm{~V}$ & $15.92 \%$ & $\mathrm{BO} \cap \mathrm{Bg}$ & $1.08 \%$ \\
$\mathrm{Bg}$ & $1.399 \%$ & $\mathrm{Gh} \cap \mathrm{V}$ & $1.98 \%$ \\
$\mathrm{~A} \cap \mathrm{BO}$ & $0.649 \%$ & $\mathrm{Gh} \cap \mathrm{Bg}$ & $0.5 \%$ \\
$\mathrm{~A} \cap \mathrm{V}$ & $26.40 \%$ & $\mathrm{~V} \cap \mathrm{Bg}$ & $3.30 \%$ \\
\hline
\end{tabular}

The table 2 illustrates the occupancy rates of the stable zones (simple classes) which reaches the rate of $54.40 \%$ and that of the zones of change (composed classes) which reaches $45.60 \%$. From the table 2, we note that the Argan class (A) and Vegetation class (V) have known a great change compared to the other classes, indeed, we found for the composed class $\mathrm{A} \cap \mathrm{V}$ a rate of change of $26.40 \%$.

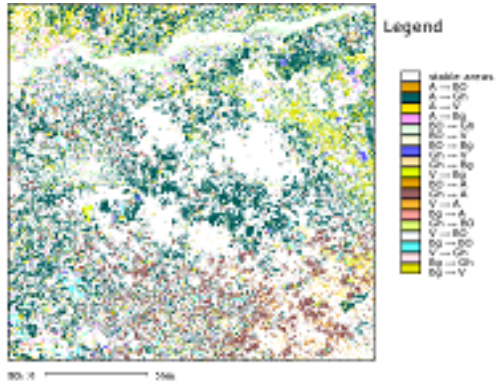

(a)

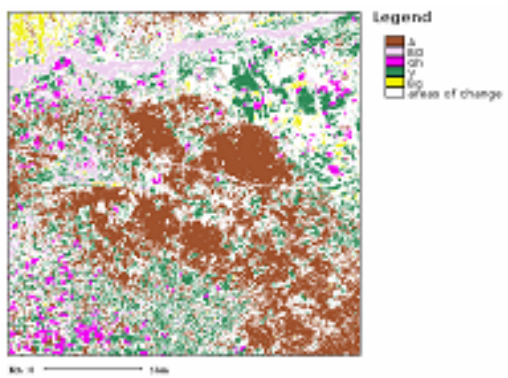

(b)

Fig. 4. Post-processed map of change zones (a) and stable zones map (b) obtained from fusion map 
From the stable zones map figure 4-(a), we note that the zone of Oued Souss (BO), the zone of the urban areas along the road Agadir-Taroudant (BO), the international airport of Almassira (BO), the parcels of the Ouled teima region (V), the greenhouses (Gh) of the Biogra region and also some surface of argan (A) are all well detected as stable zones and are assigned to simple classes. This attribution is well justified because some zones are built and are unchangeable by nature.

In the post-processed fusion map figure 4-(b), areas that have known major changes are the Argan class which becomes bare ground or vegetation, which is well explained because of the deforestation. As result we found a rate of 18.95 of the Argan class changed to vegetation in 2005.

3.3.3.2 Validation per Spectral Signature of the Results. For this spectral evaluation, we have compared the spectral signatures of the some area of changes for the two images by taking in account the distributions of spectral signatures of different themes.

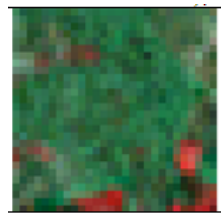

a.1

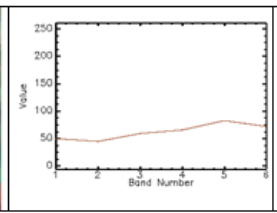

a. 2

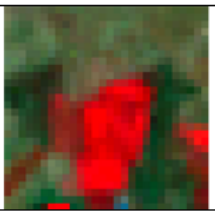

b.1

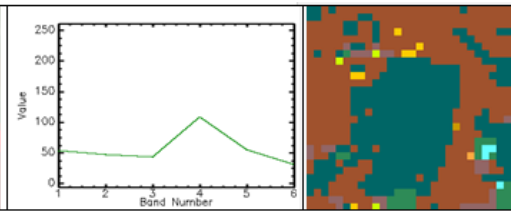

b. 2

Fig. 5. Spectral signature of the Argan class (a.2) (ETM+2002 a.1) and of the Vegetation class (b.2) (ETM+ 2005 b.1), extract of the fusion map(c)

From the figure 5, we note that the extract area has known a change of Argan theme (Class A) to vegetation theme (class V), what is shown by the change of the pixels spectral signature of the extract area which had in 2002 a spectral signature of the Argan (Class A), and became in 2005 that of the vegetation (Class V). The table 5 summarizes all the changes detection and figure 6 shows the associated postprocessed map of the fusion.

Table 3. Rate of change obtained by fusion between LANDSAT ETM+ 2002 and ETM+ 2005

\begin{tabular}{llllll}
\hline Class & \multicolumn{1}{c}{$\begin{array}{c}\text { Number of } \\
\text { pixels }\end{array}$} & \multicolumn{1}{c}{$\begin{array}{c}\text { Occupancy rate } \\
(\boldsymbol{\%})\end{array}$} & \multicolumn{1}{c}{ Class } & $\begin{array}{c}\text { Number of } \\
\text { pixels }\end{array}$ & $\begin{array}{c}\text { Occupancy } \\
\text { rate (\%) }\end{array}$ \\
\hline $\mathrm{A}$ & 104,542 & 29.0394 & $\mathrm{Gh} \rightarrow \mathrm{V}$ & 3,112 & 0.8644 \\
$\mathrm{BO}$ & 17,538 & 4.8717 & $\mathrm{Gh} \rightarrow \mathrm{Bg}$ & 471 & 0.1308 \\
$\mathrm{GH}$ & 11,458 & 3.1828 & $\mathrm{~V} \rightarrow \mathrm{Bg}$ & 2,662 & 0.7394 \\
$\mathrm{~V}$ & 57,320 & 15.9222 & $\mathrm{~V} \rightarrow \mathrm{A}$ & 26,889 & 7.4692 \\
$\mathrm{Bg}$ & 5,048 & 1.4022 & $\mathrm{Bg} \rightarrow \mathrm{A}$ & 2,439 & 0.6775 \\
$\mathrm{~A} \rightarrow \mathrm{BO}$ & 797 & 0.2214 & $\mathrm{Gh} \rightarrow \mathrm{BO}$ & 915 & 0.2542 \\
$\mathrm{~A} \rightarrow \mathrm{V}$ & 68,202 & 18.9450 & $\mathrm{~V} \rightarrow \mathrm{BO}$ & 502 & 0.1394 \\
$\mathrm{~A} \rightarrow \mathrm{Bg}$ & 4,295 & 1.1931 & $\mathrm{Bg} \rightarrow \mathrm{BO}$ & 647 & 0.1797 \\
$\mathrm{BO} \rightarrow \mathrm{Gh}$ & 4,650 & 1.2917 & $\mathrm{~V} \rightarrow \mathrm{Gh}$ & 4,005 & 1.1125 \\
$\mathrm{BO} \rightarrow \mathrm{V}$ & 25,587 & 7.1075 & $\mathrm{Bg} \rightarrow \mathrm{Gh}$ & 1,324 & 0.3678 \\
$\mathrm{BO} \mathrm{Bg}$ & 3,227 & 0.8964 & $\mathrm{Bg} \rightarrow \mathrm{V}$ & 12,831 & 3.5642 \\
\hline
\end{tabular}




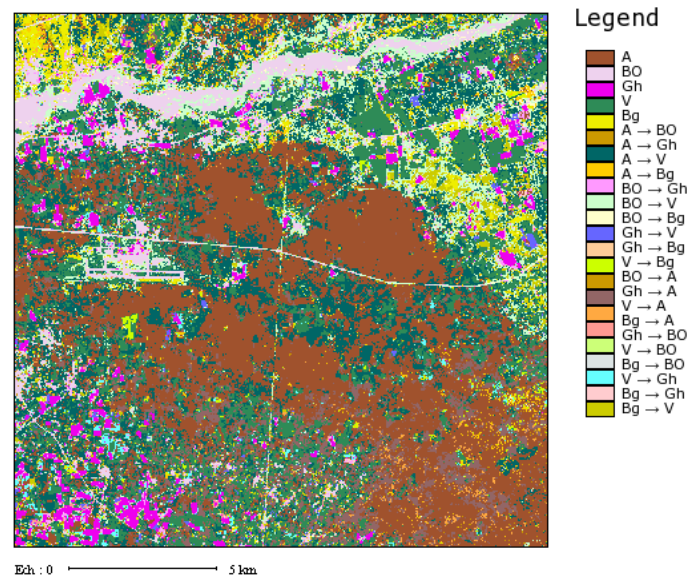

Fig. 6. Post-processed fusion map

\section{Conclusion}

In this paper we have proposed a new method for land cover changes detection. In first, we have included the spatial information in the process of fusion/classification by using a hybrid DSmT model with the introduction of contextual information using ICM classification with constraints, the use jointly of DSmT and ICM improves performance of the changes detection in terms of accuracy and exactitude. Secondly, we have proposed a new decision rule that has shown its performance and allowed us to overcome the limitations of rules decision based on the maximum generalized belief functions which are increasing and unsuited to the decision of the union and the intersection of elements. The application of this method for the cartography of the land cover changes is promising.

Acknowledgments. This work was funded by CNRST Morocco and CNRS France Grant under Convention CNRST CRNS program SPI09/11.

\section{References}

1. Smarandache, F., Dezert, J. (eds.): Advances and Applications of DSmT for Information Fusion (Collected works), vol. 1. American Research Press, Rehoboth (2004)

2. Smarandache, F., Dezert, J.: Advances and Applications of DSmT for Information Fusion (Collected works), vol. 2. American Research Press, Rehoboth (2006)

3. Smarandache, F., Dezert, J.: Applications and Advances of DSmT for Information Fusion (Collected works), vol. 3. American Research Press, Rehoboth (2009)

4. Idbraim, S., Ducrot, D., Mammass, D., Aboutajdine, D.: An unsupervised classification using a novel ICM method with constraints for land cover mapping from remote sensing imagery. International Review on Computers and Software (I.RE.CO.S.) 4(2) (2009) 
5. Ducrot, D.: Méthodes d'analyse et d'interprétation d'images de télédétection multi-sources Extraction de caractéristiques du paysage, Habilitation thesis, France, Décembre 1 (2005)

6. Mercier, G.: Outils pour la télédétection opérationnelle, Habilitation thesis, Rennes I university, France, March 2 (2007)

7. Corgne, S., Hubert-Moy, L., Dezert, J., Mercier, G.: Land cover change prediction with a new theory of plausible and paradoxical reasoning. In: ISIF 2003, Colorado, USA (March 2003)

8. Moraa, B., Fourniera, R.A., Foucherb, S.: Application of evidential reasoning to improve the mapping of regenerating foreststands. International Journal of Applied Earth Observation and Geoinformation (2010)

9. Basse, R.M.: Université de Nice, La prise en compte de l'incertitude dans une démarche de modélisation prédictive. In: MoDyS, Lyon, France, Novembre 8-9 (2006)

10. Bouakache, A., Belhadj-Aissa, A.: Satellite image fusion using Dezert-Smarandache theory, DSmT-book3, Master Project Graduation, University Houari Boumediene (2009)

11. Khedam, R., Bouakache, A., Mercier, G., Belhadj-Aissa, A.: Fusion multidate à l'aide de la théorie de Dempster-Shafer pour la détection et la cartographie des changements: application aux milieux urbain et périurbain de la région d'alger. Télédétection 6(4), 359-404 (2006)

12. Djiknavorian, P.: Fusion d'informations dans un cadre de raisonnement de DezertSmarandache appliquée sur des rapports de capteurs ESM sous le STANAG 1241, Memory to obtain the degree (M.Se.), Laval University, Quebec (2008)

13. Anne-Laure, J., Martin, A., Maupin, P.: Gestion de l'information paradoxale contrainte par des requêtes pour la classification de cibles dans un réseau de capteurs multi-modalités. In: SCIGRAD 2008, Brest, France, Novembre 24-25 (2008)

14. Foucher, S., Germain, M., Boucher, J.M., Bénié, G.B.: Multisource Classification Using ICM and Dempster-Shafer Theory. IEEE Transaction on Instrumentation and Measurement 51(2) (April 2002)

15. Bloch, I.: Fusion d'informations en traitement du signal et des images. In: IC2, Hermès Science, Traité IC2, Paris, France (2003)

16. Lemeret, Y., Lefevre, E., Jolly, D.: Fusion de données provenant d'un laser et d'un radar en utilisant la théorie de Dempster-Shafer. In: MAJECSTIC 2004, France (2004)

17. Fiche, A., Martin, A.: Bayesian approach and continuous belief functions for classification. In: LFA, Annecy, France, November 5-6 (2009)

18. Germain, M., Boucher, J.M., Bénié, G.B., Beaudry, E.: Fusion évidentielle multi source basée sur une nouvelle approche statistique floue. In: ISIVC 2004, Brest, France (2004)

19. Martin, A.: Fusion de classifieurs pour la classification d'images sonar. In: RNTI-E 2005, pp. 259-268 (Novembre 2005)

20. Chitoub, S.: Combinaison de classifieurs: une approche pour l'amélioration de la classification d'images multisources multidates de télédétection. Télédétection 4(3), 289-301 (2004) 